\title{
Alexandra Ludewig
}

The University of Western Australia

\section{Isaac Steinberg in Australien \\ Der Traum von einer jüdischen Kolonie in West- und Nordaustralien}

In den 1930er Jahren war die in London ansässige Freeland League for Jewish Territorial Colonisation (Freiland-Liga der Jüdischen Kolonisation) verzweifelt auf der Suche nach einem neuen Zuhause für die verfolgten Juden Europas. Der Schriftführer der Freeland League, Dr. Isaac Steinberg, hatte dabei "vast undeveloped areas of the world" (Steinberg 1948: 7) im Visier und fand Gefallen an Australien, insbesondere an einer Landparzelle in der westaustralischen Kimberley Region. Aus dieser historischen Konstellation ergab sich eine unerwartet ambivalente Situation für die beiden Protagonisten aus Europa und Übersee - waren es doch zwei Bevölkerungsgruppen, die in spezifischer Weise von Vertreibung und Entwurzelung betroffen waren: die durch das Naziregime verfolgten Juden Europas und die Aborigines, deren Existenzrecht in der Kimberley Region Westaustraliens als Folge dieses Umstandes zur Disposition stand. Während die bisherige Forschung sich zumeist mit der Dokumentation von Steinbergs Plänen sowie jüdischen und australischen Reaktionen darauf begnügte (vgl. Hooper 1991, Alroey 2011, Lawrence 2014, The Kimberley Society 2016), nimmt dieser Beitrag erstmals die Situation der indigenen Bevölkerung mit in den Fokus und ist damit Teil eines größeren Projektes, welches sich kritisch mit der westaustralisch-deutschen Siedlungsgeschichte auseinandersetzt (Ludewig 2016).

Der folgende Artikel wirft ein Schlaglicht auf einen historisch außergewöhnlichen Moment im zwanzigsten Jahrhundert, als ein humanitär ausgerichtetes jüdisches Siedlungsprojekt im Ergebnis eine weitere Zurückdrängung der indigenen Bevölkerung der East Kimberley-Region bedeutet hätte. Zuerst wird die Genese von Steinbergs geplanter jüdischer Kolonie in Australiens Norden nachgezeichnet, bevor das Unterfangen unter dem Gesichtspunkt des stetig anwachsenden Forschungsgebietes zum Settler Colonialism kritisch beleuchtet wird. In Anlehnung an die Verfechter des Eliminationsgedankens, allen voran die scharf formulierten Thesen von Tony Barta (1987) und Patrick Wolfe (2006), wird für den Steinberg-Plan abgeleitet, dass auch dieser zumindest das Potential zu einem ambivalenten, im Kern als Entwurzelungsstrategie ausdeutbaren Kolonisationsprojekts für jüdische Flüchtlinge in Übersee hatte. Denn für die jüdische Gruppe hätte diese Ansiedlung höchstwahrscheinlich Sicherheit vor Völkermord bedeutet, wohingegen es für die indigene Bevölkerung eine potentielle Gefahr weiterer Diskriminierung und Zurückdrängung durch Enteignung eingeläutet hätte, mit der erhöhten Wahrscheinlichkeit von Völkermord durch ebendiese Vertreibung und Enteignung in direkter Konsequenz. Patrick Wolfes Aussage "land is life" (2006: 387) trifft in diesem Fall nicht nur für die einheimische Bevölkerung der Kimberleys zu, sondern auch für die jüdischen Flüchtlinge. Es zeigt sich aber auch, dass die Umkehrung "kein Land, kein Überleben" gravierende, ubiquitäre Konsequenzen implizierte. 
Isaac Nachman Steinberg wurde 1888 in Dünaburg im russischen Zarenreich (heute Teil von Lettland) in eine jüdisch-orthodoxe, jiddisch-sprachige Familie geboren. In seiner Jugend war er ein aktives Mitglied der sozialrevolutionären Partei (Alroey 2011: 19). Aufgrund seines Aktionismus wurde er verhaftet und nach Sibirien ins Exil geschickt, ehe ihm als 19-Jährigem die Flucht nach Zürich gelang. Bald zog er von der Schweiz weiter nach Deutschland, wo er im Jahre 1910 an der Universität Heidelberg sein Doktorat in Rechtswissenschaften mit Schwerpunkt auf den Rechtsvorschriften des Talmuds abschloss. Darauf kehrte er nach Moskau zurück, wo er sich als "a leading figure in the non-Marxist Left-Social Revolutionary Party" profilierte (Rovner 2014: 155). Nach der Oktoberrevolution von 1917 wurde Steinberg Lenins erster Justizminister und somit Teil von dessen Koalitionsregierung. Nach Steinbergs Rücktritt glückte es ihm nicht, Lenins politischem Terror zu entkommen. Er wurde zweimal verhaftet, bevor ihm 1923 die Flucht nach Deutschland gelang. Gemeinsam mit seiner Frau und seinen zwei Kindern ließ er sich in Berlin nieder (vgl. Alroey 2011: 19). Unter der wachsenden nationalsozialistischen Bewegung in Deutschland fand sich Steinberg als jüdischer Intellektueller in der Gruppe der unerwünschten Oppositionellen wieder. Schon kurz nach ihrer Machtübernahme versuchten die Nazis Steinberg im Jahr 1933 zu inhaftieren, doch hielt er sich zu dieser Zeit gerade im Rahmen einer Vortragsreihe in London auf. In einer Nacht- und Nebelaktion gelang auch seiner Familie die Flucht nach England (Rovner 2014). Dort gewann Steinberg rasch an Bedeutung, sowohl im britischen PEN - "Poets, Essayists and Novelists", einer weltweiten Vereinigung von Schriftstellern - als auch in der damals größten jüdischen territorialen Organisation, der Freiland-Liga, deren Komitee er im Jahr 1935 beitrat.

Über zehn Jahre hinweg versuchte Steinberg Unterstützung für eine “Jewish Colony" zu gewinnen und in der East Kimberley Region West- und Nordaustraliens Land zu erwerben, das die damaligen Besitzer, unter ihnen die aus Irland stammende Durack-Familie, veräußern wollte. ${ }^{1}$ Das zum Verkauf stehende Land sollte 50.000 bis 75.000 jüdischen Siedlern ein neues Zuhause bieten. Obwohl die überwiegende Mehrheit der jüdischen Flüchtlinge aus städtischen Gebieten kam und wenig bis gar keine landwirtschaftlichen Kenntnisse hatte, war das so genannte "Kimberley Scheme" im Wesentlichen als landwirtschaftliches Unterfangen konzipiert. ${ }^{2}$ Steinberg teilte sein Bestreben, aus Städtern Farmer zu machen, mit vielen Zionisten des späten neunzehnten und frühen zwanzigsten Jahrhunderts, und sah darin die Möglichkeit, die osteuropäischen und russischen Juden aus ihren Ghettos zu befreien, in denen ihnen die Bewirtschaftung von Boden verwehrt wurde.

Im Mai 1939, als Steinberg schon in seinen frühen Fünfzigern war, erreichte er die westaustralische Hafenstadt Fremantle und war hochmotiviert, das Gebiet im äußersten Norden zu erkunden, das mit 28.000 Quadratkilometern in etwa die Größe von Belgien hat und fast identisch ist mit dem gesamten Bereich, der heute als Ord

1 Der in London ansässige australische Journalist Charles Henry Chomley versuchte bei den Engländern Auswanderlust nach Nordaustralien zu wecken und machte so auch die Freeland League auf das Land aufmerksam. "The portfolio of properties owned by Connor, Doherty and Durack was on the market, with [the politician] George Miles [...] looking for a buyer. He approached Steinberg in 1938 and 'proposed that the Freeland League create a chartered or limited company with a capital of $£ 200,000$ and purchase the land rights, the cattle and the buildings of Connor, Doherty and Durack Ltd'. When interest was shown, Miles worked with Steinberg to create a package in which the League would buy the properties, settle many thousands of refugees there, and begin the conversion to orchards and farms. That work was to be facilitated by damming the Ord River and generating hydro-electricity". The Kimberley Society (2016).

2 Der Plan war es, "[to] convert the country from a pastoral into an agricultural area". Steinberg zitiert in "Jewish Settlers for North West Australia. Dr Steinberg States The Case", Morning Bulletin, 7. Februar 1940, 6. 
River Bewässerungsprojekt bekannt ist. In Vorbereitung auf die "mass colonisation" (Steinberg 1948: 12) begab sich Steinberg, zusammen mit dem 22-jährigen Michael Durack, dessen 75 Jahre alten Vater Michael Patrick (M.P. genannt) Durack, sowie einem Agraringenieur der University of Western Australia, Dr. George F. Melville, auf eine Erkundungsreise durch die Gegend. Da die Exkursion in den kühleren und trockenen Monaten des späten [australischen] Herbstes und frühen Winters (Juni/ Juli 1939) stattfand, erwartete Dr. Steinberg harte Bedingungen, war aber stattdessen angenehm von den milden Konditionen überrascht, "a country that was alive" vorzufinden. ${ }^{3}$ Seine Bemerkung "alive" liest sich eher als eine Anspielung auf Fauna

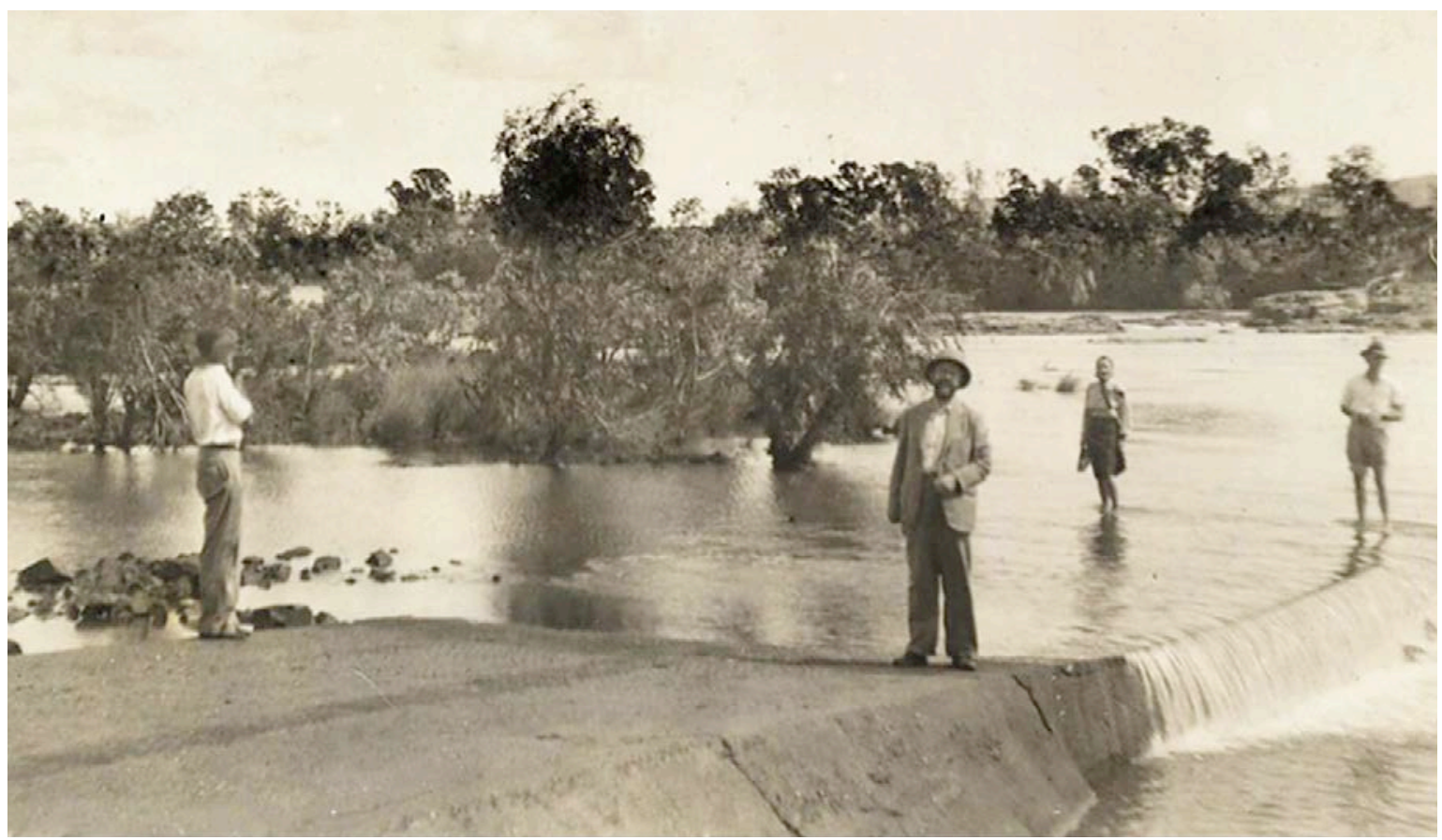

Abb. 1 - Dr. Steinberg am Ord River (1939) CElizabeth Durack (Album 1936-1939)

und Flora als ein Hinweis auf die Bewohner des Landes. Das Projekt war auf einem Teil des Landes geplant, der ursprünglich den lokal ansässigen Aborigines gehörte und wo auch weiterhin Aborigines-Gruppen (insbesondere die Miriwoong, Kadjerong, Malngin, Kija, Gurindji und Ngarinman) lebten, die nun vor allem auf der Farm der Duracks arbeiteten. Steinberg nahm dies nicht ausdrücklich zur Kenntnis, obwohl ihm ihre Anwesenheit und ihr bedeutender Beitrag zur Wirtschaft in dem Gebiet bewusst gewesen sein mussten. Steinberg kommentierte explizit, dass er viele "Weiße" in der Bevölkerung, unter ihnen insbesondere Frauen, getroffen und befragt hatte. ${ }^{4}$ Seine Formulierung verrät deutlich, dass er über die Anwesenheit von "Nicht-Weißen" Bescheid wusste; diese Tatsache wird auch von zahlreichen Fotos bestätigt, die entstanden, als Steinberg und Melville das Land erkundeten, auf dem damals 70.000 Rinder gehalten wurden. Nun sollte es eine mögliche Heimat für numerisch ebenso viele Flüchtlinge aus Europa zur Verfügung stellen. Der Konkurrenzkampf zwischen Ortsansässigen und Neuankömmlingen um Ressourcen und Arbeit würde in der Folge stark zunehmen. Eine Auflage der westaustralischen Regierung war es zudem, dass an den vielen neuen Schulen in der Kolonie nur vom

3 "It was winter here now and yet we found [...] a country that was alive" (Steinberg 1948: 18),

4 Steinberg "saw and questioned many of the white folk living there, particularly the women" (1948: 23). 
Bildungsministerium in Perth anerkannte Lehrpersonen und andere aus weiter entfernten Verwaltungszentren, die wesentlichen Dienstleistungen für die neuen Siedler bereitstellen dürften (Steinberg 1948: 11); die Aborigines des East Kimberley wurden von Steinberg auch in diesem Zusammenhang nicht erwähnt.

Obwohl den Ureinwohnern dieses Landes heutzutage große Teile der Fläche als Besitz zuerkannt werden, standen die Ansprüche der Aborigines auf das Land 1939 weder auf der australischen Rechtsordnung noch auf der Tagesordnung der von Dr. Steinberg geführten Erkundungstruppe (Gettler 1993: 143). Trotz der Erfahrungen seines eigenen Volkes in Bezug auf Vertreibung und Umsiedlung, Vernichtung und Ausrottung schien Steinberg seine Augen vor der Existenz, geschweige denn der Notlage der einheimischen Bevölkerung zu verschließen. Steinberg wies vielmehr darauf hin, dass auch andere Migrantengruppen bereits erfolglos versucht hatten, sich im äußersten Norden von Westaustralien anzusiedeln, und beschrieb Wyndhams "deserted streets with its tiny abandoned Chinese and Afghan shops" (Steinberg 1948: 17). Er verweist zudem auf die potentiellen Handelspartner jenseits der Meere: "[...] a splendid harbour giving ready access to the entire world and particularly to the uncounted coloured peoples of Asia" (ibid.).

Diese wohl kalkulierten Überlegungen kontrastierten mit der Tatsache, dass er den traditionellen Bewohnern des Landes, das er bereiste, keinerlei Beachtung schenkte. Stattdessen verwies Steinberg in seiner Vision nicht nur die Duracks des Landstriches; sondern verbannte sowohl andere Kolonisten wie auch Ureinwohner aus seinem Siedlungskonzept, wenn er sinnierte: "What could not Jewish colonists achieve here!" (Steinberg 1948: 17).

Es entsprach seiner idealisierten Vorstellung von jüdischer Siedlungskolonisation, dass diese erfolgreicher $\mathrm{zu}$ werden verspreche als die anderer Volksgruppen, was er am Beispiel der erfolgreichen Erschließung von Wüstenregionen in Palästina (ibid.: 10) bestätigt sah. Steinberg hatte die Vision, dass das Potential dieses "schlafenden" (21) oder "schlummernden" (22) Landes im Norden Australiens unter jüdischer Bearbeitung erstmals voll ausgeschöpft würde, und benutzte unwissentlich die gleiche ideologische Rechtfertigung für die gewünschte Besiedlung wie die kolonialen Siedler, die zuvor die Auslöschung der indigenen Völker bewusst einkalkuliert hatten; "that 'we' could use the land better than they [the original and subsequent owners, i.e. in this scheme's case the Duracks and the local Aboriginal peoples]" (Wolfe 2006: 389).

East Kimberley versprach unter jüdischem Pflug und Management eine wirtschaftlich produktive und gesellschaftlich prosperierende Region $\mathrm{zu}$ werden. ${ }^{5}$ Steinberg argumentierte hier in Richtung terra nullius, da er von einem bislang nicht urbar gemachten und noch nicht kultivierten Landstreifen ausging.

Abgesehen von dieser impliziten Referenz wurde den lokalen Aborigines, trotz ihrer immer noch großen Anzahl und ihrer tief verankerten Traditionen (vgl. Curthoys 2014: 210ff.), kein Platz in Steinbergs Überlegungen eingeräumt. Es gibt weder Anhaltspunkte dafür, dass Steinberg eine Assimilationspolitik verfolgte, noch dahingehend, die Frage zu beantworten, ob er die Aborigines bewusst oder unbewusst

5 Mein Dank geht an John Docker, der mich auf Waswo (1997) hingewiesen hat. Waswo bezieht sich auf die in der Aeneis genannte Legende, in der jene, die als Kolonisatoren kamen, sich selbst als Kulturträger im Besitz von selbst ernanntem überlegenem Wissen in Bezug auf Landwirtschaft, Städtebau, Recht und Religion, sehen (siehe auch Docker 2008). 


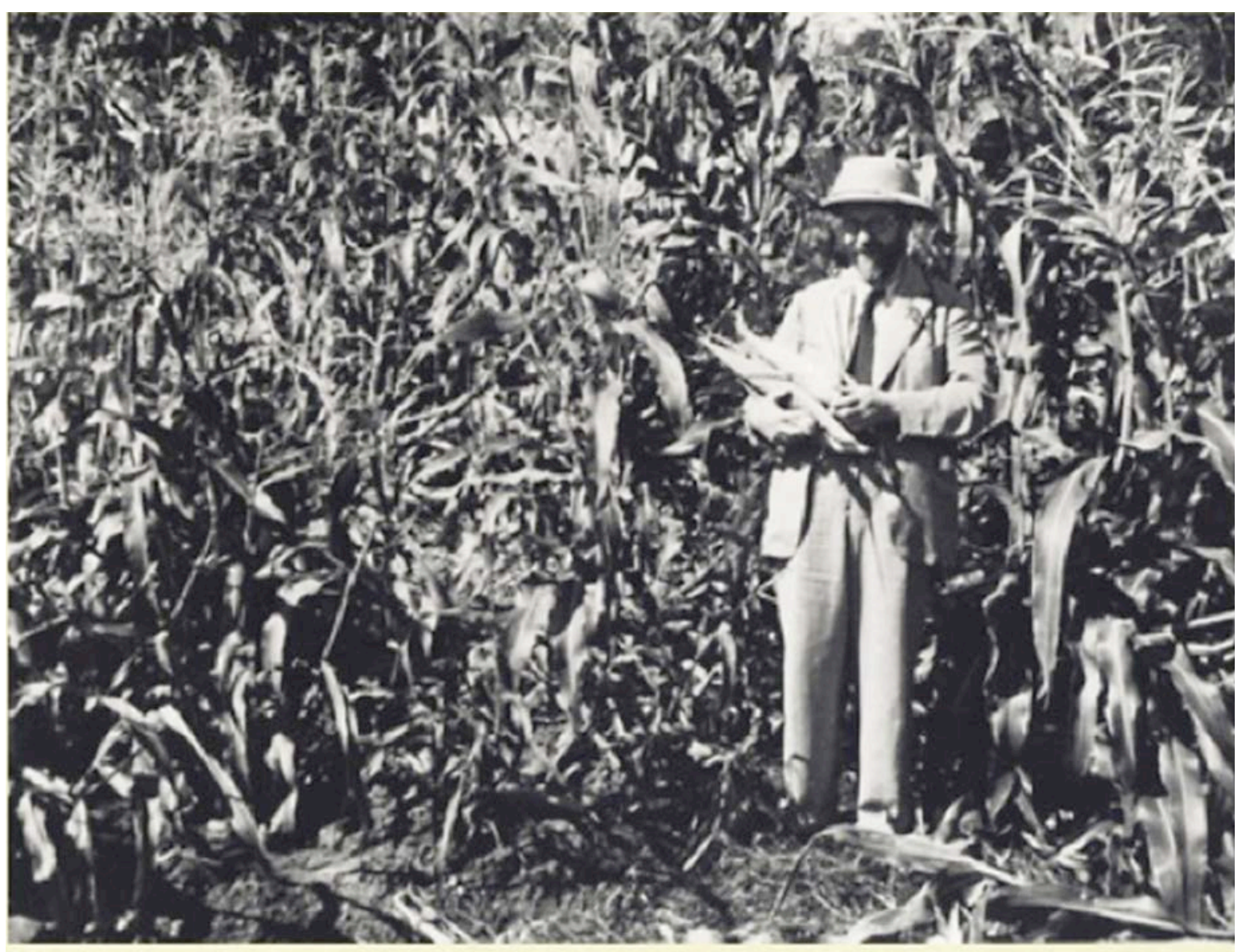

\section{Dr Steinberg inspecting crops}

Abb. 2 - Begutachtung der Ernte - Argyle/Durack Estate (1939)

CElizabeth Durack (Album 1936-1939)

unsichtbar machte, denn physisch blieben sie, wie auch die Fotos belegen, präsent. Das gleiche Paradox der 'abwesenden Anwesenden' gilt auch für den Schriftverkehr, den Steinberg mit den Duracks und der Regierung unterhielt. Das Schicksal der Ureinwohner wurde aus der Debatte ausgeschlossen. Die Aborigines selbst erhielten keine Gelegenheit zur Stellungnahme. Dieses Verhalten war nicht ungewöhnlich, berücksichtigt man die weit verbreitete Einstellung der Kolonialisten gegenüber den Ureinwohnern. Exemplarisch führt der Anthropologe und Historiker Patrick Wolfe aus: "Aborigines were accorded no rights to their territory, informal variants on the theme of terra nullius being taken for granted in settler culture" (2006: 391). Dies illustrierend wurde auch die Besetzung des Landes durch die Familie Durack von Mary Durack so beschrieben, als ob ihre Vorfahren in "a deserted camp" eingezogen seien (Durack 1959: 217). Nur an einem Punkt in ihrer Niederschrift lässt sie ein wahrheitsgetreueres Abbild der Wirklichkeit durchscheinen. Im Hinblick auf die wachsende Kritik an gewalttätigen Ausschreitungen (vor allem nach den Massakern in der Forrest River Mission und dem Ernest River Massaker im Jahr 1926, vgl. Gribble 1928) schrieb sie von der nahezu greifbaren Alternative, mit der die Siedler konfrontiert waren: "Were they to admit defeat, clear out and give the country back to the Aborigines [...]?" (Durack 1959: 313). Es ist historiographisch gut dokumentiert, welche Einstellung die traditionellen Bewohner im Allgemeinen ge- 


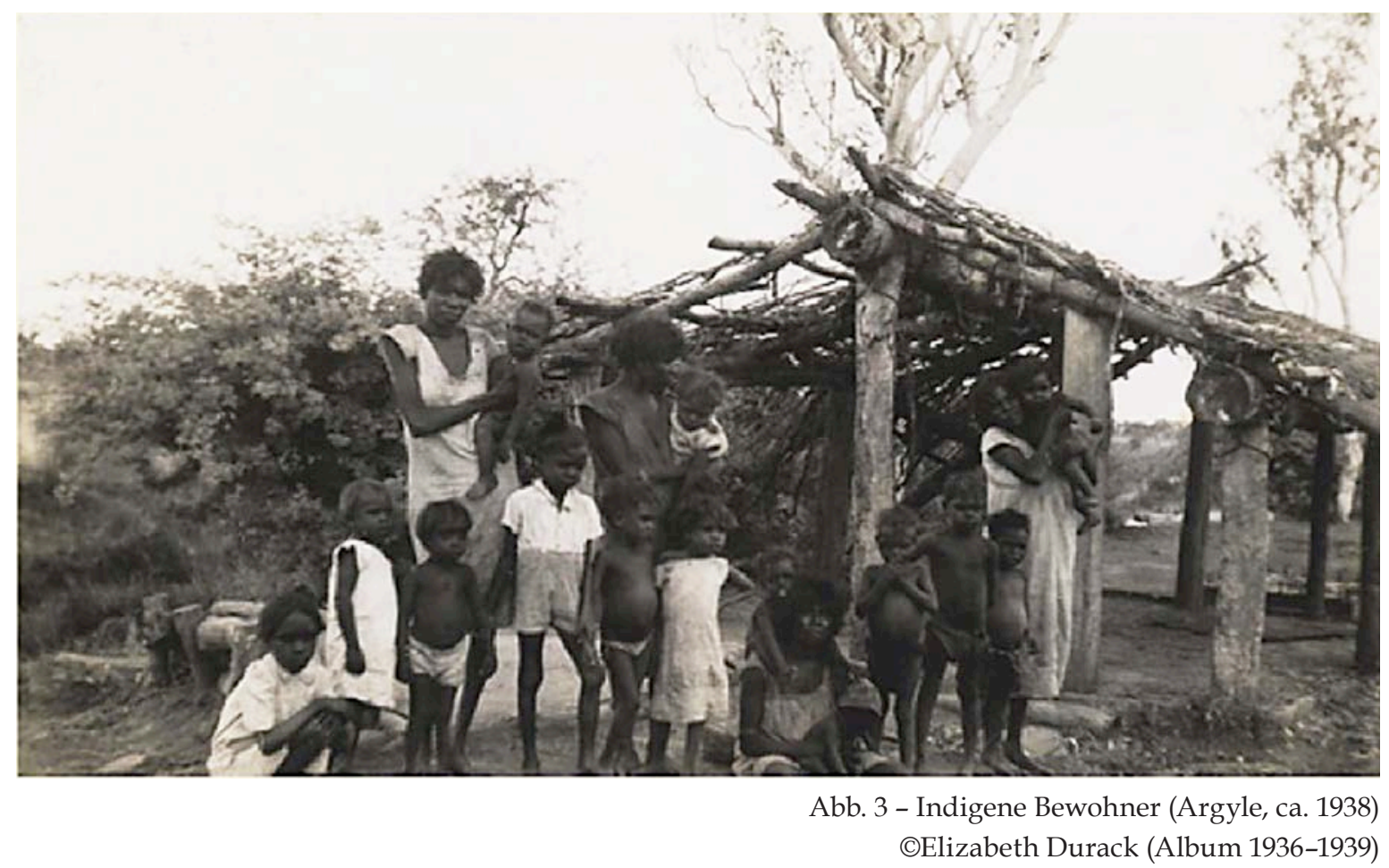

genüber der Kolonisierung hatten, seit die europäischen Entdecker und Siedler im neunzehnten Jahrhundert in ihre Gebiete vorgedrungen waren. Die Forschungen von Henry Reynolds und Noel Loos über die Gewalt in Grenzgebieten, ${ }^{6}$ zuletzt in Reynolds' nuancierten Arbeiten zu Forgotten War (2013) und Unnecessary Wars (2016) dokumentiert, liefern genügend Beweise für die Auseinandersetzungen zwischen den Kolonialisten und den Aborigines, denn, in der Tat, "[...] the Kimberley tribes fought a long and savage war against the pastoralists" (Green 1981: 116).

Als Steinberg im Jahr 1939 in die Kimberley-Region kam, kommentiert er staunend: "It is an empty, spacious, sleeping land" (Steinberg zitiert 1948 im Sydney Morning Herald). Der Begriff "sleeping" ist eine sehr verräterische Wortwahl, da sie auf Steinbergs grundlegende Idee zurückgeht, dass allein die richtige(n) Person(en), also jene aus der jüdischen Diaspora, den schöpferischen Geist des Landes, d. h. sein landwirtschaftliches Ertragspotential, aus seinem Dornröschenschlaf erwecken und befreien müsste(n). Betrachtet man die heutige Besiedlungsdichte, erscheint es nicht weniger naiv. Zur gleichen Zeit sieht Steinberg in diesem "almost mysterious land" (Steinberg 1948: 14), diesem "land of mystery" (15), diesem "magic land" (17) etwas seltsam Vertrautes, wohl auch deshalb, weil er dieser Landschaft einige der Merkmale seines Heimatlandes sowie seiner Wahlheimat(en) zuschrieb. Er dachte zurück an Russland, als er Landstriche als "endless savanna" oder "steppe" beschrieben hatte, und fand den Boden "not unlike the Russian chernoziom, 'black earth"' (Steinberg 1948: 19). Er erkannte darin auch englische Landschaften wieder: "From time to time we came upon thickets of trees, plants and grass so fragrant and cheerful that I could have fancied myself on some exquisitely cultivated English estate" (ibid.). Offensichtlich sah er das, was er sehen wollte, etwas sehr Vertrautes und Angenehmes, und wünschte sich, den Besitz dieses Landes für sich selbst als ideal zu betrach-

6 Reynolds fokussierte auf das Schicksal der indigenen Bevölkerung, z. B. in Aborigines and Settlers: The Australian Experience, 1788-1939 (1972); als auch in The Other Side of the Frontier. Aboriginal Resistance to the European Invasion of Australia (1981); Frontier. Aborigines, Settlers and Land (1987); Why Weren't We Told? (2000); An Indelible Stain? The Question of Genocide in Australia's History (2001) und vielen weiteren seiner Publikationen. 
ten. Auf der einen Seite versuchte Steinberg, durch diese Beschreibungen das Land an seine gewohnte Umgebung bzw. sein gewohntes Umfeld narrativ anzugleichen und zu zeigen, dass er und andere europäische Juden auf einzigartige Weise in der Lage wären, das Land produktiv zu machen. Immer wieder macht er in diesem Zusammenhang indirekte Anspielungen darauf, dass dies weder von den Aborigines, noch von den Duracks erreicht worden sei. Auf der anderen Seite musste er Investoren und zukünftige Siedler davon überzeugen, dass das Land vielversprechend war, ausreichend fruchtbar und ertragreich. In seinem Buch über die Entwicklung der Siedlerkulturen beschreibt James Belich ähnliche Beschönigungen, die in der Auswanderungsliteratur des neunzehnten Jahrhunderts zu finden sind, als "boosterism" (2009: 85). Vivian Bickford-Smith diskutiert am Beispiel von Kapstadt die sogenannte "place selling"-Literatur und die narrativen und rhetorischen Konventionen, die in imperialen Texten benutzt wurden, um Kolonien zu fördern (2012: 133-151). Als solches greift Steinberg eine metaphorische Redensart des neunzehnten Jahrhunderts auf und beweist, dass dies auch im zwanzigsten Jahrhundert noch eine lohnenswerte Strategie für den jüdischen Kolonialisierungsdiskurs darstellt.

Das von Bickford-Smith beschriebene Phänomen der "Einzigartigkeit" lässt sich auch in Steinbergs Beschreibung wiederfinden. Steinberg bewirbt das von ihm explorierte Land als auf einzigartige Weise für das Siedlungsprogramm geeignet: “[...] this land has no savage beasts or venomous snakes, and that it knows no dangerous diseases" (Steinberg 1948: 22). Dies ist eine bemerkenswert hoffnungsvolle Aussage, da ein wenig Nachforschung das Gegenteil bewiesen hätte, und es wirft die Frage auf, ob Steinberg absichtlich falsch informiert wurde oder selbst die Absicht hatte, jenen, die er hier ansiedeln wollte, eine beschönigende Auskunft zu geben. Bei Steinbergs eigener Überquerung des Ord River hätte er Salzwasser-Krokodile vorfinden müssen (Durack Web) sowie die in diesem Gebiet zahlreichen einheimischen Giftschlangen. Zudem gab es auch Malaria, Dengue-Fieber, Lepra und Tuberkulose, um nur einige der gefährlichen Infektionskrankheiten zu nennen, deren Auswirkungen auf den Menschen in der Region bekannt waren. ${ }^{7}$ Viehkrankheiten sollen hier erst gar nicht erwähnt werden. Alfred Crosby erläutert diese Form des Siedlerkolonialismus mit dem Begriff "ökologischer Imperialismus" 8 - denn auch in der Vergangenheit hatten Hoffnungsvolle vielerorts Land in Besitz genommen und gleichfalls betont, dass ihre Wahl- und Wunschorte frei von Krankheiten seien und eine günstige Umgebung böten. Die dann jedoch in der Folge eingeführten Krankheiten hatten schnell dramatische Auswirkungen auf die dort lebenden Menschen, die örtliche Fauna und Flora. Da manche neu kolonisierten Siedlungszonen klimatisch Europa sehr ähnlich waren, setzten sich zudem schnell europäische Landwirtschaftsformen und Viehzuchtrassen vor Ort durch; und dies oft auf Kosten der Ureinwohner und ihrer Kultur.

Während Steinberg beschönigend die indigene, nur scheinbar ungefährlich anmutende Fauna und Flora romantisierte, alle Bedenken über das Klima ${ }^{9}$ beiseite

7 Die Mutter von M.P. starb an Malaria. Lepra und Grippeviren wurden unter anderem im Zuge der weiBen Besiedlung von asiatischen Arbeitern eingeführt und auf die indigene Bevölkerung übertragen, die keine Abwehr dagegen hatte.

8 Australien war für diese Begrifflichkeit geeignet, während die meisten Teile Afrikas und Asiens bereits ihre speziellen Formen der Landwirtschaft und ihre endemischen Krankheiten hatten, wodurch sie oft weniger einladend für die Europäer waren und groß angelegte Siedlungskolonien verhindert wurden. Nordaustralien galt im Gegensatz dazu als ein unbeschriebenes Blatt (vgl. Crosby 1986).

9 Die Region gehört zu den heißesten in Australien und unterliegt dẹn Auswirkungen verheerender Wetterextreme: der Dürre, gewaltigen tropischen Wirbelstürmen und Überschwemmungen. 
schob und das Erntepotential der Region in seinem Bericht bewusst oder aufgrund falscher Informationen unbewusst als viel zu hoch ansetzte, zeigte die Bereitwilligkeit der Duracks, das Land für nur $£ 180.000$ zu verkaufen, ${ }^{10}$ eine offensichtliche Kehrseite der Medaille. Dennoch ignorierte Steinberg die historische Überlieferung der von ihnen erfahrenen harten Prüfungen, sowie Probleme und Missgeschicke in der Region. Die anfängliche Besitzergreifung des Landes durch die Duracks erfolgte zu einem hohen Preis, sowohl für die lokale indigene Bevölkerung (Reynolds 1972; 1981; 2001) als auch für die Neuankömmlinge. Ein Rückblick illustriert dies: Im Juli 1882 schiffte sich eine Gruppe, die von M.P. Duracks Onkel - einem weiteren Michael - angeführt wurde, ausgestattet mit Pferden, Proviant und zwei Aborigine-Viehzüchtern aus Queensland, in Brisbane ein. Sie segelten zum Cambridge Gulf und King Sound, mit der Absicht, große Landstriche um den Ord River in Besitz zu nehmen.

From Thylungra station Michael's brother Patrick, 'Patsy' Durack, organised the droving of 7250 head of breeding cattle and 200 horses on a 3000-mile (4828 $\mathrm{km}$ ) trek, the longest undertaken by Australian drovers up to that time. They reached the Ord River in two years and four months with a loss of half the cattle and several men; the venture cost some $£ 72,000$. In 1886 [Patsy] Durack's two elder sons [M.P. and John] went by sea and set up Argyle station on the Behn River. (Durack 1972)

Im selben Jahr, als die Duracks im Zuge ihrer Pionierarbeit - oder, wie Tom Stannage es nannte, ihres "taking land"-Projektes (1985: 8) - weiter in die East Kimberley Region vordrangen, verloren sie ihren Cousin John (1849-1886), der von einem Speer getötet worden war (Anonymous 1886: 3). Ein ähnliches Schicksal ereilte ein anderes Familienmitglied. Jeremiah (1853-1901), der die Rosewood und Dunham Farmen gegründet hatte, wurde von lokalen Aborigines ermordet, als er auf seiner Veranda schlief. Mary Durack, eine der Töchter von M.P., hielt mehrere blutige Begegnungen schriftlich fest und beschrieb, wie sich belesene, gebildete Männer in rachsüchtige Viehzüchter verwandelten: "[...] once in the Kimberley they all more or less subscribed to the philosophy of "us or them"' (1959: 314). Die darauf folgende Periode offenbarte ein Bild unfriedlicher Koexistenz oder, laut Marys Schwester Elizabeth Durack, eines der "mutual exploitation" (1935: 25). Dieser Umstand führte u. a. dazu, dass jenen Aborigines, die auf der Rinderfarm ${ }^{11}$ arbeiteten, westliche Namen gegeben wurden, während Elizabeth, wie im Rahmen der Aufdeckung einer der berüchtigtsten Kunstbetrügereien dokumentiert wurde, sowohl Motive der Aborigines für ihre Bilder entlehnte als auch eine indigene Identität annahm (vgl. Durack Clancy 2009; Morrison 2009 sowie Snell 2009). Die Tatbestände der strukturellen Kolonisation erklären auf beispielhafte Weise die immer wiederkehrenden, oftmals banal anmutenden Handlungsprozesse, die im Ergebnis den Siedlerkolonialismus ausmachen und, nicht selten, die Tendenz zur Auslöschung indigener Völker beinhalten, ${ }^{12}$ obwohl die Duracks den Ruf hatten, besser als andere Arbeitgeber zu sein. Nun, da das Land ihnen seinen Dienst erwiesen hatte, stand eine Veräußerung desselben im Vordergrund, keineswegs eine Rückgabe an seine ursprünglichen Eigentümer - die Aborigines.

10 Sie waren - zum Zeitpunkt des Kaufangebots - nach Jahren der Dürre massiv verschuldet.

11 Ob die Arbeit freiwillig war, oder ob es sich dabei um ein "system of forced labour on pastoral stations" handelte, ist weiterhin Gegenstand von Diskussionen (Green 1998: 449).

12 Tony Barta würde diese Vorgehensweise als "Völkermord" bezeichnen, da Völkermord für ihn eine notwendige Folge der Einrichtung von Siedlungskolonien ist (1987: 237-251; vgl. auch Docker 2014: 74-89). 
In den späten 1930er Jahren waren die Duracks daran interessiert, sich im Rahmen eines lukrativen Geschäftes von dem erschlossenen Land zu trennen, und Steinberg hatte den Eindruck, dass "[i]t is not to be presumed that Australians or Englishmen will be prepared in the near future to settle in these regions, they do not seem to have the nineteenth-century urge to open up new lands" (Steinberg 1948: 22). Als ob die Haltung der kolonialen Siedler eine Angelegenheit der vergangenen Jahrhunderte wäre, versah Steinberg die geplante Übernahme des Landes durch sein Volk mit einer anderen moralischen und historischen Qualität - einer Qualität, die sich nur in seiner Sicht von den Absichten und Gewohnheiten früherer Kolonialisten unterschied. Er betrachtete die erwartete jüdische Besiedlung des Landes nicht als Landnahme und somit als eine Fortsetzung der strukturellen und systemischen Bedrohung für das Wohlergehen der Indigenen, für die jegliche Form des Landverlustes eine Frage des Überlebens darstellte. Da Steinberg die noch zahlenstarke einheimische Bevölkerung der Region nicht erwähnt, auch nicht im Hinblick auf ihre mögliche zukünftige Arbeitskraft, kann es sein, dass er angenommen hatte, dass der Prozess der Verdrängung und der Kolonisierung bereits von den Duracks durchgeführt worden war, so dass nach dieser Auslegung die jüdische Landnahme den indigenen Menschen nicht mehr schaden könnte, da diese bereits zuvor - wenn nicht "eliminiert", wie Wolfe es nannte - so doch zumindest unsichtbar gemacht worden seien, nicht zuletzt als Folge früherer Enteignung. Für Steinberg war also das Kimberley-Siedlungsprogramm nicht darauf ausgerichtet, die Menschen vor Ort zu integrieren, oder - wie in anderen Kolonialutopien - zu "zivilisieren" und zu missionieren (Osterhammel 2009). Steinberg träumte vermeintlich nur von einer besseren Nutzung des Landes, sowohl in Bezug auf das Erntepotential wie auch interessanterweise im Hinblick auf die von der australischen Politik oftmals diskutierte mangelnde Wehrhaftigkeit des Kontinents.

In seiner Lobbyarbeit für die jüdische Massenansiedlung in der East Kimberley Region machte Steinberg sich in seiner Rhetorik eine lang gehegte australische Angst zu Nutze: Dabei ging es um die Theorie, entweder den Kontinent zu besiedeln oder ihn zu verlieren, und dem damit verbundenen Wunsch, den Norden des Landes zu bevölkern, wobei Steinberg auch an die australische Solidarität appellierte und eine humanitäre Geste für das jüdische Volk forderte. Mit dieser doppelgleisigen Argumentation glaubte er Unterstützung für seine geplante Besiedlung von einer Vielzahl politischer und sozialer Gruppen zu gewinnen; in ihren Kreisen fanden sich mehrere Professoren der University of Western Australia, z.B. Fred Alexander und Walter Murdoch, sowie der Chefredakteur von The West Australian. Walter Murdoch, zum Beispiel, fühlte sich veranlasst, eine Stellungnahme für die Lokalzeitung zu schreiben und sich für das Programm einzusetzen: "On every ground, moral and material, I say that we should support the project with enthusiasm" (1939: 7). Murdoch prognostizierte, dass die Kolonie auch andere Bevölkerungsgruppen in die Region locken und ihre wirtschaftliche Entwicklung unterstützen würde; in diesem Teil des Landes kultivierte die öffentliche Meinung seit vielen Jahren die Angst, vom Norden her durch eine asiatische Invasion verwundbar zu sein.

Wichtig zu erwähnen ist auch, dass der zur Perth Hebrew Congregation gehörende 70-jährige Rabbiner D.I. Freedman, der in Ungarn geboren und in England ausgebildet worden war, ebenfalls Steinbergs Idee befürwortete. Steinberg wurde demnach in gleicher Weise von der kleinen jüdischen Gemeinde unterstützt, die 
speziell im Zuge der verschiedenen Goldrauschzeiten in Westaustralien gegen Ende der 1880er Jahre deutlich gewachsen war. Die meisten Juden in Westaustralien stammten aus Großbritannien und Südafrika, während wenige aus Palästina kamen. Die jüdische Community Westaustraliens stand in starkem Kontrast zu den dominierenden jüdischen Stimmen auf der östlichen Seite Australiens. Dies zeigte sich in besonderer Weise mit Blick auf eventuelle Erwägungen einer jüdischen Masseneinwanderung: Hier waren letztere eher zwiegespalten, da sie keine Aufmerksamkeit als religiöse Minderheit auf sich ziehen wollten. Schließlich wurde die vorherrschende Gesellschaft Australiens als sehr homogen wahrgenommen, und der Versuch, sich davon abzuheben, wurde im Allgemeinen von der australischen Gesellschaft nicht positiv aufgenommen.

So war es durchaus überraschend, dass Steinbergs Idee von der Regierung Westaustraliens Ende 1939 befürwortet wurde. Allerdings kam von der Seite der Bundesregierung in Canberra zuerst keine definitive Unterstützung. Als Dr. Steinberg im Oktober 1939 von Perth nach Melbourne reiste, war der Krieg bereits ausgebrochen, wodurch seine Rückkehr nach Europa verhindert und weitere Korrespondenzen mit der Regierung unter Robert Menzies unbedeutend wurden, da diese sich auf wichtigere Dinge konzentrieren musste. Es dauerte bis zum 1. Februar 1941, bevor die australische Regierung offiziell bekannt gab, ihre Entscheidung bezüglich des Vorschlages aufzuschieben: "[...] the present was not an appropriate time to give consideration to the matter".13 Als solches existierte der Plan, mit dem man jüdischen Flüchtlingen tatsächlich noch hätte helfen können, nur für einen Zeitraum von etwas mehr als einem Jahr; dieser wurde letztlich auf Eis gelegt und im Jahr 1943 durch die anschließende Regierung unter Premierminister John Curtin kategorisch abgelehnt, wobei es zu diesem Zeitpunkt bereits vier Jahre zu spät gewesen wäre, um den europäischen Juden Zuflucht zu gewähren. Steinbergs eigene Mutter verstarb, wie so viele andere europäische Juden, in Auschwitz. Die indigene Bevölkerung der East Kimberley Region entkam hingegen vermutlich einer erneuten Verdrängung von ihrem angestammten Land, und ihr langer historischer Weg zur Anerkennung der eigenen Landrechte mag sich auf diese Weise verkürzt haben.

Damit legte die historische Konstellation der alliierten Verpflichtungen Australiens im Zweiten Weltkrieg die grotesk anmutende Versuchung und menschlich verzweifelte Hoffnung nahe, ein humanitär ausgerichtetes jüdisches Siedlungsprojekt umzusetzen, das im Ergebnis die Zurückdrängung der indigenen Bevölkerung der East Kimberley-Region bedeutet hätte. Steinbergs Traum von einer jüdischen Kolonie im Norden Australiens hatte zumindest das Potential zu einem ambivalenten, im Kern als Entwurzelungsstrategie ausdeutbaren Kolonisationsprojekt jüdischer Flüchtlinge in Übersee.

\section{Danksagung}

Mein Dank gilt Shino Konishi und John Docker, die einen früheren Entwurf kommentierten und so entscheidend dazu beigetragen haben, dass dieser Artikel aussa- 
gekräftiger wurde. Außerdem danke ich Stephanie Schimkowitsch, Johannes Brunnschweiler und Iris Ludewig-Rohwer für sprachliche wie inhaltliche Anregungen.

Die Genehmigung zur Veröffentlichung der Photos aus dem Photoalbum von Elizabeth Durack (Formative Years II: 1936-1939) erfolgte mit freundlicher Genehmigung von Perpetua Durack Clancy (Curator/Executor - the Estate of Elizabeth Durack, Nov. 2017): http://www.elizabethdurack.com/

\section{Literaturverzeichnis}

Adhikari, Mohamed, ed., 2014. Genocide on Settler Frontiers: When Hunter-Gatherers and Commercial Stock Farmers Clash. Cape Town: UCT Press.

Alroey, Gur, 2011. "'Zionism without Zion'? Territorialist Ideology and the Zionist Movement, 1882-1956", Jewish Social Studies: History, Culture, Society, n.s. 18, no. 1, 1-32.

Anonymous, 1886. "Murder of John Durack by Natives at Kimberley", Northern Territory Times and Gazette, 11 December, 3.

Anonymous, 1940. "Jewish Settlers for North West Australia. Dr Steinberg States The Case", Morning Bulletin, 7 Feb, 6.

Anonymous, 1948. "Wasted Years", Sydney Morning Herald, 16 October, 6.

Barta, Tony, 1987. "Relations of Genocide: Land and Lives in the Colonization of Australia", in: I. Wallimann and M.N. Dobkowski, eds., Genocide and the Modern Age: Etiology and Case Studies of Mass Death, 237-251.

Belich, James, 2009. Replenishing the Earth: The Settler Revolution and the Rise of the AngloWorld, 1783-1939. Oxford: Oxford University Press.

Bickford-Smith, Vivian, 2012. "Providing Local Colour? 'Cape Coloureds', 'Cockneys', and Cape Town's Identity from the Late Nineteenth Century to the 1970s", Journal of Urban History, 38 (1), 133-151.

Crosby, Alfred W., 1986. Ecological Imperialism. The Biological Expansion of Europe 900-1900. New York: Cambridge University Press.

Curthoys, Ann, 2014. "Indigenous Dispossession and Pastoral Employment in Western Australia During the Nineteenth Century: Implications for Understanding Colonial Forms of Genocide", in: Mohamed Adhikari, ed., Genocide on Settler Frontiers: When Hunter-Gatherers and Commercial Stock Farmers Clash, 210-231.

Docker, John, 2008. The Origins of Violence: Religion, History and Genocide. Sydney: UNSW Press.

Docker, John, 2014. "A Plethora of Intentions: Genocide, Settler Colonialism and Historical Consciousness in Australia and Britain", The International Journal of Human Rights, Dec., 74-89.

Durack, Elizabeth, 1936-1939. “Photo Album. Formative Years", Web. 2 Feb 2017. <http:// www.elizabethdurack.com/photo_album.php?img_id $=112 \&$ album_id $=26 \&$ curPage $=0>$.

Durack, Mary and Elizabeth, 1935. All-about: The Story of a Black Community on Argyle Station Kimberley. Sydney: The Bulletin.

Durack, Mary, 1959. Kings in Grass Castles. Sydney: Bantam.

Durack, Mary, [1972] 2017. "Durack, Patrick (1834-1898)", Australian Dictionary of Biography, National Centre of Biography, Australian National University, Web. 1 Feb 2017. $<$ http://adb.anu.edu.au/biography/durack-patrick-3457/text5281>.

Durack Clancy, Perpetua, 2009. “Eddie Burrup - A Daughter's View”, Westerly, Vol. 54(1), 72-76.

Gettler, Leon, 1993. An Unpromised Land. Fremantle, WA: Fremantle Arts Centre Press.

Green, Neville, 1981. "Aborigines and White Settlers", in: C. Tom Stannage, ed., A New History of Western Australia, 72-123.

Green, Neville, 1998. "From Princes to Paupers. The Struggle for Control of Aborigines in Western Australia 1887-1898", Early Days. Journal of the Royal Western Australian Historical Society, Vol. 11, Pt. 4, 446-462. 
Gribble, Reverend, 1928. "Royal Commission into the Killing and Burning of Bodies of Aborigines in East Kimberley, and into Police Methods When Effecting Arrest", Vol. 1, West Australian Proceedings of Parliament and Papers 1928, in: Sharmane Stone, ed., Aborigines in White Australia, 149-151.

Hooper, Beverley, 1991. “The Unpromised Land: A Jewish Refugee Settlement in the Kimberley?", Aspects of Ethnicity, 12, 85-94.

The Kimberley Society, “Tropical Agriculture Proposals \& Endeavours - 1836-1941”, 2016. http://www.kimberleysociety.org/images/kimbsoc---iujobohshe.pdf.

Lawrence, Dashiel, 2014. "To no Avail. Supporters and Opponents of the Kimberley Scheme", Melbourne Historical Journal, Vol. 42 (1), 197-228.

Ludewig, Alexandra, 2016. Born German, Re-Born in Western Australia. Selected Stories 18412016. Crawley: UWAP.

Morrison, Louise, 2009. “The Art of Eddie Burrup", Westerly, Vol. 54, 1, 77-83.

Mossenson, David, 1990. Hebrew, Israelite, Jew: The History of the Jews of Western Australia. Perth: UWAP.

Murdoch, Walter, 1939. “Our Opportunity. A Home For Refugees", The West Australian, 15 July, 7.

Osterhammel, Jürgen, 2009. Kolonialismus. Geschichte, Formen, Folgen. München: C.H. Beck.

Reynolds, Henry, 1972. Aborigines and Settlers: The Australian Experience, 1788-1939. North Melbourne: Cassell Australia.

Reynolds, Henry, 1981. The Other Side of the Frontier. Aboriginal Resistance to the European Invasion of Australia. Townsville: Department of History and Politics, James Cook University.

Reynolds, Henry, 1987. Frontier. Aborigines, Settlers and Land. Sydney: Allen \& Unwin.

Reynolds, Henry, 2000. Why Weren't We Told? Ringwood: Viking.

Reynolds, Henry, 2001. An Indelible Stain? The Question of Genocide in Australia's History. Ringwood: Viking.

Reynolds, Henry, 2013. Forgotten War. Sydney: New South Books.

Reynolds, Henry, 2016. Unnecessary Wars. Sydney: New South Books.

Rovner, Adam L., 2014. In the Shadow of Zion. Promised Lands Before Israel. New York: NYU Press.

Snell, Ted, 2009. “The Fantasist: Elizabeth Durack and Eddie Burrup", Westerly, Vol. 54(1), $85-87$.

Stannage, C. Tom, ed., 1981. A New History of Western Australia. Perth: UWAP.

Stannage, C. Tom, 1985. Western Australia's Heritage. The Pioneer Myth. Nedlands: University Extension.

Steinberg, Isaac N., 1948. Australia - The Unpromised Land. In Search of a Home. London: Victor Gollancz Ltd.

Stone, Sharmane, ed., 1974. Aborigines in White Australia. Melbourne: Heinemann.

Wallimann, I., and M. N. Dobkowski, eds., 1987. Genocide and the Modern Age: Etiology and Case Studies of Mass Death. Westport, CT: Greenwood Press.

Waswo, Richard, 1997. The Founding Legend of Western Civilization: From Virgil to Vietnam. London: University of New England Press.

Wolfe, Patrick, 2006. "Settler Colonialism and the Elimination of the Native", Journal of Genocide Research, 8 (4), 387-409. 